\section{Verhältnis qualifikationsgebundener Abrechnungsgenehmigungen zu den Fachgebietsgrenzen}

\section{Einleitung}

Die Frage nach dem Verhältnis qualifikationsgebundener Abrechnungsgenehmigungen (gelegentlich auch „Fachkundenachweis“ oder „Fachkundegenehmigung“ genannt) zu den Grenzen des ärztlichen Fachgebiets ${ }^{1}$ war in den letzten Jahren bereits mehrfach Gegenstand der sozialgerichtlichen Rechtsprechung. ${ }^{2}$ Nicht selten ging es dabei um die Frage, ob ein Facharzt für Diagnostische Radiologie, dem eine Genehmigung zur Ausführung und Abrechnung von Leistungen nach dem Einheitlichen Bewertungsmaßstab (EBM) ${ }^{3}$ auf Grundlage der Vereinbarung für Strahlendiagnostik und -therapie erteilt worden ist, entsprechende Leistungen in der vertragsärztlichen Versorgung auch tatsächlich erbringen und gegenüber der Kassenärztlichen Vereinigung (KV) abrechnen darf.

Das Verhältnis qualifikationsgebundener Abrechnungsgenehmigungen zu den Fachgebietsgrenzen war zudem bereits Gegenstand früherer Beiträge in der Rubrik Radiologie und Recht ${ }^{4}$, dennoch fehlt es dieser Problematik nicht an Aktualität. Unlängst hatte sich das

Im Interesse der Lesbarkeit haben wir auf geschlechtsbezogene Formulierungen verzichtet. Selbstverständlich sind immer w/m/d gemeint, auch wenn explizit nur eines der Geschlechter angesprochen wird.

1 Unter der neuen MWBO 2018 (hierzu in diesem Beitrag unter Ziffer 3. und 5.) können innerhalb eines Gebietes auch mehrere Facharztbezeichnungen erworben werden, während „[d]ie Gebietsdefinition [...] die Grenzen für die Ausübung der fachärztlichen Tätigkeit [bestimmt]“ (§2 Abs. 2 S. 2 MWBO 2018). Dies hat im Rahmen der in diesem Beitrag angeführten Rechtsprechung noch keine Rolle gespielt. An der dortigen Ausdrucksweise wird im Rahmen der hiesigen Darstellungen grundsätzlich festgehalten („Fachgebiet“ etc.).

2 Z. B. BSG, Urteil vom 04.05.2016, Az.: B 6 KA 13/15 R, MedR 2017, 179 ff.; Urteil vom 08.08.2018, Az.: B 6 KA 47/17 R, MedR 2019, $164 \mathrm{ff}$, abrufbar in juris.

3 Aktuelle Fassung mit Stand vom 3. Quartal 2021 (Wirkung ab 01.07.2021).

4 Zuletzt Wigge/Kirsch in: RöFo 2021, 733, 735; zuvor Wigge/Schütz in: RöFo 2017, $171 \mathrm{ff}$.
Bundessozialgericht (BSG) in einer aktuellen Entscheidung ${ }^{5}$ erneut damit zu befassen. Der Senat hat darin seine vorbestehende Auffassung in dieser Angelegenheit bestätigt und erforderliche Abgrenzungskriterien zwischen wesentlichen Fallkonstellationen weiter herausgearbeitet und präzisiert. Aus diesem Anlass widmen wir uns in diesem Beitrag den sich aus der Rechtsprechung des BSG ergebenden Grundsätzen zu den Fragestellungen, einerseits, unter welchen Voraussetzungen eine qualifikationsgebundene Abrechnungsgenehmigung zu erteilen ist und andererseits, wie sich diese zu den weiterbildungsrechtlichen fachärztlichen Gebietsgrenzen verhält.

\section{Die qualifikationsgebundene Abrechnungsgenehmigung}

\section{a. Qualitätssicherungsvereinbarun- gen nach § 135 Abs. 2 SGB V}

Den Ausgangspunkt der Betrachtung bildet die Vorschrift des $\S 135$ Abs. 2 SGB V. Diese gestattet den Partnern der Bundesmantelverträge, d. h. der Kassenärztlichen Bundesvereinigung (KBV) und dem Spitzenverband Bund der Krankenkassen (auch: GKVSpitzenverband),

„[f]ür ärztliche und zahnärztliche Leistungen, welche wegen der Anforderungen an ihre Ausführung oder wegen der Neuheit des Verfahrens besonderer Kenntnisse und Erfahrungen (Fachkundenachweis), einer besonderen Praxisausstattung oder anderer Anforderungen an die Versorgungsqualität bedürfen, [...] einheitlich entsprechende Voraussetzungen für die Ausführung und Abrechnung dieser Leistungen [zu] vereinbaren."

(§135 Abs. 2 S. 1 SGB V, sämtliche Hervorhebungen im Beitrag durch die Verfasser.)

Auf Grundlage dieser gesetzlichen Vorschrift haben die Partner des Bundesmantelvertrags verschiedene Vereinbarungen für den Bereich der vertragsärztlichen

5 BSG, Beschluss vom 17.03.2021, Az.: B 6 KA $27 / 20 \mathrm{~B}$, abrufbar in juris.

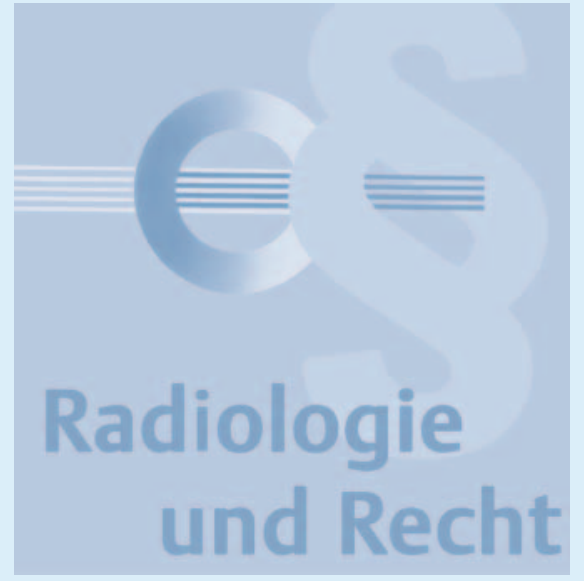

Versorgung geschlossen. Da die Versorgungsqualität - wie gezeigt - das maßgebliche Motiv für den Abschluss derartiger Vereinbarungen ist, werden diese auch als „Qualitätssicherungsvereinbarungen“ bezeichnet. Sie sind dem Bundesmantelvertrag als Anlagen beigefügt.

Am Beispiel der Vereinbarung zur Strahlendiagnostik und -therapie ${ }^{6}$ in der seit dem 01.10.2020 geltenden Fassung (StrahlendiagV) wird im Folgenden gezeigt, wie sich die Qualitätssicherungsvereinbarungen nach § 135 Abs. 2 SGB V zu anderen für die ärztliche Berufsausübung maßgeblichen Regelungskomplexen, vornehmlich dem ärztlichen Weiterbildungsrecht, verhalten.

Die StrahlendiagV regelt die Voraussetzungen für die Ausführung und Abrechnung von Leistungen der diagnostischen Radiologie, der Strahlentherapie und der Nuklearmedizin in der vertragsärztlichen Versorgung (§ 1 S. 1 StrahlendiagV). Die Erbringung und Abrechnung solcher Leistungen bedarf der Genehmigung durch die KV (§2 S. 1 StrahlendiagV), die zu erteilen ist, wenn der Vertragsarzt die Voraussetzungen hierfür erfüllt. In der StrahlendiagV wird dabei zwischen zwei Kategorien der Erteilungsvoraussetzungen unterschieden: solche der fachlichen Befähigung, niedergelegt in Abschnitt B StrahlendiagV, und solche der apparativen Ausstattung, niedergelegt in Abschnitt C StrahlendiagV (vgl. § 2 S. 2 StrahlendiagV).

6 Vereinbarung von Qualifikationsvoraussetzungen gemäß § 135 Abs. 2 SGB V zur Durchführung von Untersuchungen in der diagnostischen Radiologie und Nuklearmedizin und von Strahlentherapie, abrufbar unter: https://www. kbv.de/media/sp/Strahlendiagnostik.pdf. 
Der Arzt muss der KV gegenüber nachweisen, dass er für die Methode bzw. das Verfahren, für das er die entsprechende Genehmigung begehrt, sowohl über die erforderliche fachliche Befähigung als auch die vorgeschriebene apparative Ausstattung verfügt (vgl. § 3 S. 1 StrahlendiagV).

\section{b. Anforderungen an die fachliche Befähigung}

Für die hiesige Betrachtung indes sind allein Voraussetzungen der fachlichen Befähigung nach der StrahlendiagV von Bedeutung. Diese sind für verschiedene radiologische Untersuchungsverfahren sowie nuklearmedizinische und strahlentherapeutische Verfahren im Einzelnen vorgegeben. Die Anforderungen, die an die fachliche Qualifikation eines Arztes gestellt werden, der beabsichtigt, in der vertragsärztlichen Versorgung z. B. Leistungen der Computertomographie (CT) auszuführen und abzurechnen, finden sich in $§ 7$ StrahlendiagV; hinsichtlich strahlentherapeutischer Leistungen enthält $\S 9$ StrahlendiagV die einschlägigen Regelungen.

\section{Bedeutung der Fachgebiets- grenzen im Verhältnis zu den Voraussetzungen der Quali- tätssicherungsvereinbarungen gemäß § 135 Abs. 2 SGB V}

Die StrahlendiagV knüpft insoweit hinsichtlich der fachlichen Befähigung des Arztes unter anderem an bestimmte Facharztbezeichnungen an und lässt die im Rahmen einer geordneten Facharztweiterbildung erworbene fachärztliche Kompetenz nach den Vorgaben der jeweiligen Weiterbildungsordnung (WBO), ausdrücklich auch in der vertragsärztlichen Versorgung, im Wesentlichen als ausreichende fachliche Qualifikation genügen. Jedoch ist erkennbar, dass es einem Vertragsarzt - jedenfalls nach der Konzeption der StrahlendiagV auch möglich sein soll, die Abrechnungsgenehmigung zu erlangen, ohne die hierfür erforderliche, in der Vereinbarung genannte Facharztweiterbildung absolviert zu haben. Diese Vorgaben müssten jedoch mit den Fachgebietsgrenzen ärztlicher Tätigkeit nach den Heilberufs- und Kammergesetzen der Länder ${ }^{7}$ und den Weiterbildungsordnungen der Landesärztekammern vereinbar sein.

In der Muster-Weiterbildungsordnung 2018 in der aktuell geltenden Fassung vom 26.06.2021 (MWBO 2018) der Bundesärztekammer (BÄK) und den Weiterbildungsordnungen der Landesärztekammern heißt es in $\S 2$ Abs. 2 S. 2, dass die Grenzen für die Ausübung der fachärztlichen Tätigkeit durch die Gebietsdefinition bestimmt werden.

Die Fachgebietsgrenzen nach der Weiterbildungsordnung beschränken somit die fachärztliche Tätigkeit. Nach der Rechtsprechung des BVerfG ist diese Beschränkung auf das Fachgebiet dem Arzt auch vor dem Hintergrund des Rechts auf freie Berufsausübung aus Art. 12 Abs. 1 S. 1 GG zumutbar,

„wenn die Facharztbereiche vom fachlich-medizinischen Standpunkt aus sachgerecht abgegrenzt sind und angenommen werden kann, daß der Facharzt in der auf sein Fachgebiet beschränkten Tätigkeit eine ausreichende Lebensgrundlage findet." 8

An die Pflicht, die Fachgebietsgrenzen nach der für ihn jeweils geltenden Weiterbildungsordnung zu beachten, ist der Vertragsarzt gebunden. ${ }^{9}$ Auf welche Fassung der Weiterbildungsordnung im Einzelfall abzustellen ist, wird in diesem Beitrag unter der Ziffer 5. näher beleuchtet.

Zwar hat das BVerfG hinsichtlich der privatärztlichen Leistungserbringung - vor dem Hintergrund des $\S 1$ Abs. 2 S. 1 der Gebührenordnung für Ärzte (GOÄ) - entschieden, dass auch eine systematische fachgebietsübergreifende Tätigkeit nicht zu beanstanden ist, solange der Anteil fachfremder Leistungen an den erbrachten Gesamtleistungen des Arztes unter $5 \%$ liegt und sich damit „noch im

7 Vgl. z. B. Nordrhein-Westfalen: §41 Abs. 1 Heilberufsgesetz NRW (HeilBerG NW), Niedersachsen: § 36 Abs. 2 Kammergesetz für die Heilberufe (HKG).

8 BVerfG, „Facharztbeschluss“, Beschluss vom 09.05.1972, Az.: 1 BvR 518/62, BVerfGE 33, 125 ff., abrufbar in juris, Rn. 128.

9 BVerfG, Nichtannahmebeschluss vom 16.07.2004, Az.: 1 BvR 1127/01, SozR 4-2500 $\S 135 \mathrm{Nr} 2$ = NZS 2005, 91 ff., abrufbar in juris, Rn. 22. geringfügigen Bereich" bewegt. ${ }^{10}$ Dies gilt jedoch angesichts der in dieser Weise höchstrichterlich entschiedenen Sachverhalte nicht für die vertragsärztliche Tätigkeit.

Unter besonderer Berücksichtigung dieser Umstände stellt sich die Frage, ob und inwieweit Vertragsärzte, die die Anforderungen der StrahlendiagV in fachlicher Hinsicht erfüllen, aber eine Facharztbezeichnung zu führen berechtigt sind, die unter $\S 9$ Abs. 1 StrahlendiagV nicht ausdrücklich genannt wird, mit der grundsätzlichen Verpflichtung zur Beschränkung ihrer ärztlichen Tätigkeit auf das eigene Fachgebiet in Konflikt geraten können.

\section{Berücksichtigung der Fachgebietsgrenzen im Rahmen der Erteilung einer qualifikationsgebundenen Abrechnungsgenehmigung}

In dem folgenden Überblick werden zu dem vorliegenden Themenkomplex relevante Entscheidungen des BSG voneinander abgegrenzt und das Verhältnis der Fachgebietsgrenzen zu den qualifikationsgebundenen Abrechnungsgenehmigungen dargestellt.

\section{a.}

Zuletzt hatte sich das BSG in diesem Jahr im Rahmen eines Beschlusses mit der Thematik zu beschäftigen. ${ }^{11}$ Dem Verfahren lag dabei folgender Sachverhalt zu Grunde:

Die Klägerin führte die Facharztbezeichnungen für Nuklearmedizin und für Diagnostische Radiologie. In der vertragsärztlichen Versorgung jedoch war sie lediglich als Fachärztin für Nuklearmedizin zugelassen. Ihr Antrag auf Erteilung der Abrechnungsgenehmigung für Leistungen der CT auf der Grundlage der StrahlendiagV (in der für den Rechtsstreit maßgeblichen Fassung) wurde von der zuständigen KV abgelehnt. Das Bayerische LSG als Berufungsinstanz bestätigte diese Ablehnung als rechtmäßig. Die Klägerin erfülle als Fachärztin für Diagnostische Radiologie

10 BVerfG, Stattgebender Kammerbeschluss vom 01.02.2011, Az.: 1 BvR 2383/10, NZS 2012, 62 ff., abrufbar in juris, Rn. 30.

11 BSG, Beschluss vom 17.03.2021, a. a. O. (Fn. 5); hierzu auch Wigge/Kirsch, a. a. O. (Fn. 4). 
zwar die fachlichen Genehmigungsvoraussetzungen nach der StrahlendiagV. Jedoch könne sie die streitgegenständlichen CT-Leistungen nicht fachgebietskonform erbringen, da sie in der vertragsärztlichen Versorgung allein als Fachärztin für Nuklearmedizin zugelassen sei. Die qualifikationsgebundene Abrechnungsgenehmigung sei in diesem Fall nicht zu erteilen, da von vornherein ausgeschlossen sei, dass die Klägerin von ihr Gebrauch machen und CT-Leistungen in der vertragsärztlichen Versorgung erbringen könne. ${ }^{12}$

Das BSG hat diese Rechtsauffassung bestätigt und die Beschwerde gegen die Nichtzulassung der Revision als unbegründet zurückgewiesen. ${ }^{13}$ Zur Begründung verweist der 6. Senat im Wesentlichen auf zwei gefestigte höchstrichterliche Rechtsprechungslinien:

Zum einen sei in dieser Fallkonstellation der Zusammenhang zwischen der weiterbildungsrechtlichen Fachgebietsgrenze und der Zulassung als Vertragsärztin entscheidungsrelevant. Hierzu stellt der 6. Senat des BSG Folgendes fest:

„Berufsrechtlich ist die Klägerin berechtigt, beide Fachgebietsbezeichnungen (Fachärztin für Nuklearmedizin und Fachärztin für Diagnostische Radiologie) zu führen. Jedoch steht diese Aussage des Senats nicht für sich allein, sondern es wird sogleich ein Bezug zu der Tätigkeit des Arztes als Vertragsarzt hergestellt. So betont der Senat stets, dass Beschränkungen des Fachgebiets den Arzt auch in seiner Tätigkeit als Vertragsarzt erfassen [...]. Selbst ein Arzt, der berufsrechtlich mehrere Gebietsbezeichnungen führen darf, aber nur für ein Fachgebiet in der vertragsärztlichen Versorgung zugelassen ist, muss sich im Rahmen der vertragsärztlichen Versorgung auf Leistungen des Gebietes beschränken, für das er zugelassen ist. " 14

Hieraus ergibt sich, dass neben die Berechtigung zur Führung der in den Qualifikationsvereinbarungen nach $\S 135$ Abs. 2 SGB V

12 BSG, Beschluss vom 17.03.2021, a. a. O. (Fn. 5), Rn. 1; vgl. Bayerisches LSG, Urteil vom 15.07.2021, Az.: L 12 KA 12/18.

13 BSG, Beschluss vom 17.03.2021, a. a. O. (Fn. 5), Rn. 3.

14 BSG, Beschluss vom 17.03.2021, a. a. O. (Fn. 5), Rn. 8 m.w.N. ausdrücklich genannten Facharztbezeichnungen, auf die bspw. §9 Abs. 1 StrahlendiagV als fachliche Voraussetzung zurückgreift, die Voraussetzung hinzutritt, dass (Fach-) Ärzte auch für dasjenige Fachgebiet vertragsärztlich zugelassen sein müssen, dem die Leistungen zugehörig sind, hinsichtlich derer sie eine qualifikationsgebundene Abrechnungsgenehmigung begehren.

Zum anderen trifft der Senat deutliche Aussagen zum Verhältnis von qualifikationsgebundener Abrechnungsgenehmigung und der Verpflichtung des Arztes, seine ärztliche Tätigkeit innerhalb der Grenzen seines Fachgebiets auszuüben:

Grundsätzlich sei zwischen der Erteilung von Abrechnungsgenehmigungen und der Frage, ob der Arzt die genehmigungsbedürftigen Leistungen unter Beachtung der Grenzen seines Fachgebietes tatsächlich erbringen darf, zu unterscheiden. ${ }^{15}$ Infolgedessen sei eine qualifikationsgebundene Abrechnungsgenehmigung regelmäßig zu erteilen, wenn der Arzt die entsprechenden fachlichen Voraussetzungen, die in der Qualitätssicherungsvereinbarung niedergelegt sind, erfülle. Dies gelte selbst dann, wenn die jeweilige Leistung für den Arzt fachfremd sei. ${ }^{16}$

Von diesem Grundsatz besteht jedoch nach Auffassung des Senats - eine Ausnahme. In bestimmten Fällen sei eine Abrechnungsgenehmigung, selbst bei Vorliegen aller sonstigen fachlichen Voraussetzungen nach der jeweiligen Qualitätssicherungsvereinbarung, bereits dann nicht zu erteilen, wenn die Leistungen, auf deren Ausführung und Abrechnung die qualifikationsgebundene Abrechnungsgenehmigung gerichtet ist, sich für den Arzt als fachfremd darstellten. ${ }^{17}$

Die Frage, ob in Folge fehlender Zugehörigkeit einer Leistung zum Fachgebiet des Arztes, der die Erteilung einer Abrechnungsgenehmigung begehrt, bereits deren Erteilung zu versagen oder aber (lediglich) - nach der Erteilung einer solchen Genehmigung - die tatsächliche Durchführung und Abrechnung von Leistungen im Einzelfall abzulehnen ist.

15 BSG, Beschluss vom 17.03.2021, а. а. O. (Fn. 5), Rn. 7 m.w.N.

16 Vgl. ebd.

17 Vgl. ebd. wurde durch das BSG zuletzt mit Urteil vom 15.07.2020 ${ }^{18}$ einer Klärung zugeführt.

In dem der Entscheidung zu Grunde liegenden Sachverhalt, wurde einem vertragsärztlich tätigen Facharzt für Physikalische und Rehabilitative Medizin eine Abrechnungsgenehmigung für die Durchführung sonographischer Leistungen nach der Ultraschall-Vereinbarung nicht erteilt, da diese Leistungen für ihn fachfremd und somit nicht abrechnungsfähig gewesen seien. ${ }^{19}$

Hierzu stellt das BSG fest, dass in der zu beurteilenden Situation über die Frage der Fachgebietsfremdheit einer bestimmten Leistung des Klägers schon nicht zu entscheiden gewesen sei. Als entscheidungserheblich sieht der 6 . Senat allein die Frage an, ob eine etwaige Fachfremdheit der streitgegenständlichen sonographischen Leistungen der Erteilung der beantragten Genehmigung an den Kläger überhaupt entgegenstehe. ${ }^{20}$ Der Senat kommt in den Entscheidungsgründen diesbezüglich zu einer eindeutigen Aussage:

„Ob dem LSG auch im Übrigen im Ergebnis dahingehend zu folgen ist, dass die sonographischen Leistungen für den Kläger als fachfremd einzuordnen sind, bleibt offen. [...] Denn das Argument der Fachfremdheit allein vermag im Ergebnis jedenfalls die Versagung der Genehmigung nicht zu stützen [...].“21

Im Ergebnis bedeutet dies, dass in dem dieser Entscheidung zu Grunde liegenden Sachverhalt die Fachfremdheit der Leistung, auf die sich die streitgegenständliche Abrechnungsgenehmigung nach der gemäß § 135 Abs. 2 SGB V erlassenen Ultraschall-Vereinbarung bezieht, die Erteilung dieser Genehmigung nicht ausschließt.

Der erkennbare Grund, warum die Fachfremdheit der Leistungen die Erteilung der

18 BSG, Urteil vom 15.07.2020, Az.: B 6 KA 19/ $19 R$, SozR 4-2500 §135 Nr 30, abrufbar in juris.

19 BSG, Urteil vom 15.07.2020, a. a. O. (Fn. 18), Rn. 2; LSG Saarland, Urteil vom 12.04.2019, Az.: L 3 KA 4/16, abrufbar in juris, Rn. 1 ff.

20 BSG, Urteil vom 15.07.2020, a. a. O. (Fn. 18), Rn. 28.

21 BSG, Urteil vom 15.07.2020, a. a. O. (Fn. 18), Rn. 23; vgl. auch Rn. 28. 
Abrechnungsgenehmigung in diesem Fall nicht ausschließt, ist die Möglichkeit des Arztes, von der erteilten Abrechnungsgenehmigung - wenn auch nicht systematisch, so doch wenigstens im Ausnahmefall 22 - tatsächlich Gebrauch machen zu können.

Hierzu führt das BSG Folgendes aus:

„An diesen Grundsätzen hat sich durch das Senatsurteil vom 4.5.2016 (B 6 KA 13/15R [...]) nichts geändert. Soweit der Senat dort entschieden hat, dass ein Arzt für Diagnostische Radiologie keinen Anspruch auf die Erteilung einer Genehmigung für Strahlentherapie hat, weil diese Behandlungen für ihn fachfremd sind, betraf dies eine besondere Fallgestaltung. Die Begründung der Entscheidung bezieht sich allein auf die Frage, ob ein für ein Methodenfach ( $R a-$ diologie) zugelassener Arzt unter dem Aspekt der Fachfremdheit eine Fachkundegenehmigung für die Leistungen eines anderen Methodenfaches (Strahlentherapie) erhalten kann. Fachkundebezogene Genehmigungen muss die [KV] in diesen Situationen nicht erteilen, weil ausgeschlossen ist, dass der Arzt im Rahmen der vertragsärztlichen Versorgung davon Gebrauch machen kann. Bei Ärzten, die für organbzw körperbezogene Fachgebiete (zB Gynäkologie, Orthopädie) zugelassen sind, kann dagegen kaum generell ausgeschlossen werden, dass bestimmte fachgruppenübergreifende Leistungen wie die Sonographie, die in den meisten organbezogenen Fachgebieten eingesetzt wird, zumindest in besonders gelagerten Fällen erbracht werden können, auch wenn sie typischerweise fachfremd sind. $O b$ das der Fall ist, kann und muss im Verfahren der Berichtigung nach §106 d SGB V geklärt werden (BSG Urteil vom 8.8.2018 - B 6 KA 47/ $17 R$ - SozR 4-2500 § $135 \mathrm{Nr} 27 \mathrm{RdNr} 19) .{ }^{23}$

Besteht also eine denkbare Möglichkeit für den Facharzt, in zulässiger Weise aufgrund der qualifikationsgebundenen Abrechnungsgenehmigung auch unter Berücksichtigung der Fachgebietsgrenzen Leistungen in der vertragsärztlichen Versorgung zu erbringen und abzurechnen, so ist die Genehmigung zu erteilen, selbst wenn der Arzt diese voraussichtlich nur in geringem Umfang nutzen können wird. Die Frage der

22 BSG, Urteil vom 15.07.2020, a.a. O. (Fn. 18), Rn. 32 m.w.N.

23 BSG, Urteil vom 15.07.2020, а. а. O. (Fn. 18), Rn. 33; näher erläuternd auch Rn. 34.
Nutzbarkeit der qualifikationsgebundenen Abrechnungsgenehmigung beantwortet sich unter Berücksichtigung der vorstehenden Ausführungen danach, ob der antragstellende Arzt für ein methodenbezogenes oder ein organbezogenes Fachgebiet vertragsärztlich zugelassen ist. Ein Arzt, der die Facharztbezeichnung eines Methodenfaches führt und der für dieses zugelassen ist, kann dagegen die Leistungen eines anderen Methodenfaches auch nicht ausnahmsweise erbringen und hierfür demnach schon die qualifikationsgebundene Abrechnungsgenehmigung nicht erhalten. ${ }^{24}$

\section{b.}

Die Rechtsprechung des BSG zum Verhältnis der Fachgebietszugehörigkeit einer Leistung und der qualifikationsgebundenen Abrechnungsgenehmigung lässt sich danach wie folgt zusammenfassen:

- Im Falle eines Facharztes, der einem Methodenfach angehört - wie u. a. der Facharzt für Radiologie, Nuklearmedizin, Strahlentherapie und Labormedizin - und der eine Abrechnungsgenehmigung für Leistungen beantragt, die ebenfalls ausschließlich einem anderen methodenbezogenen Fachgebiet zugeordnet sind (z. B. die Magnetresonanz-Angiographie - MRA - dem Radiologen ${ }^{25}$ ), gilt, dass die Genehmigung seitens der KV nicht erteilt werden darf. Leistungen anderer Methodenfächer, die nicht zum Weiterbildungsinhalt des betreffenden Fachgebietes gehören, stellen sich für den Vertragsarzt nicht nur als fachfremd dar, sondern dürfen von diesem - selbst im Ausnahmefall - nicht zulasten der KV erbracht und abgerechnet werden.

- Soweit die Erbringung einer Leistung durch einen Vertragsarzt, selbst unter Berücksichtigung ihrer Fachgebietsfremdheit, zumindest nicht völlig ausgeschlossen, also ausnahmsweise möglich ist, besteht nach der Rechtsprechung des BSG kein Grund, bereits die Erteilung der Abrechnungsgenehmigung zu versagen. Dies ist bei den organbezogenen Fachgebieten sowie im Falle sog. Querschnittsfächer, wie dem Fachgebiet der

24 Vgl. BSG, Urteil vom 04.05.2016, а. а. O. (Fn. 2), Rn. 22, ebenso Rn. 10 und 19.

25 Vgl. LSG Nordrhein-Westfalen, Urteil vom 15.02.2012, Az.: L 11 KA 79/10, abrufbar in juris, Rn. 40.
Physikalischen und Rehabilitativen Medizin anzunehmen, da dieses „sich weder auf ein Organsystem noch auf eine rehabilitative Strategie alleine begrenzen lässt.“26

c.

Die weiteren Konsequenzen, die sich aus der Fachfremdheit einer Leistung für den Vertragsarzt ergeben, stellt das BSG in seinem Urteil vom 15.07.202027 wie folgt dar:

- Zwischen einer qualifikationsgebundenen Abrechnungsgenehmigung, die gemäß einer Vereinbarung auf der Grundlage von $\S 135$ Abs. 2 SGB V erteilt worden ist, und der Frage danach, welche Leistungen der Arzt unter Berücksichtigung des Erfordernisses der Einhaltung der Fachgebietsgrenzen auch tatsächlich erbringen darf, ist grundsätzlich, mit Ausnahme des dargestellten Falles der Methodenfächer, zu trennen. ${ }^{28}$

- Für fachfremde Leistungen können Ärzte keine Vergütung erhalten. Erbringt ein Vertragsarzt solche Leistungen dennoch, ist die zuständige KV zur sachlich-rechnerischen Richtigstellung berechtigt. Wird einem Arzt eine Abrechnungsgenehmigung für eine bestimmte Leistung erteilt, führt dies daher nicht automatisch dazu, dass er diese Leistung, unabhängig von dem Fachgebiet, für das er zugelassen ist, im Rahmen der vertragsärztlichen Versorgung auch tatsächlich erbringen und abrechnen darf. ${ }^{29}$ Nach Ansicht des BSG eröffnet die Abrechnungsgenehmigung, auch wenn diese trotz der Fachfremdheit der Leistung erteilt worden ist, eben „nicht die Möglichkeit zur systematischen Erbringung fachfremder Leistungen [...]." ${ }^{30}$

Nach alledem ergibt sich auch für den Bereich der vertragsärztlichen Versorgung für den Facharzt ggf. eine Möglichkeit, in besonderen Situationen fachfremde Leistungen zu erbringen. Die grundsätzlich geltende Verpflichtung zur Beachtung der Fachgebietsgrenze findet ihren Niederschlag aber nicht

26 BSG, Urteil vom 15.07.2020, а. а. O. (Fn. 18), Rn. 36.

27 BSG, Urteil vom 15.07.2020, а. а. O. (Fn. 18).

28 BSG, Urteil vom 15.07.2020, а. а. O. (Fn. 18), Rn. $29 \mathrm{f}$.

29 BSG, Urteil vom 15.07.2020, а. а. O. (Fn. 189), Rn. 30.

30 Ebd. 
wie im privatärztlichen Bereich durch eine quantitative Beschränkung des (noch) zulässigen Umfangs von fachfremd erbrachten Leistungen.

Stattdessen wird in der vertragsärztlichen Versorgung eine Beschränkung im Bereich derjenigen Leistungen, für deren Ausführung und Abrechnung eine qualifikationsgebundene Genehmigung auf der Grundlage einer Qualitätssicherungsvereinbarung nach §135 Abs. 2 SGB V benötigt wird, schon auf der Ebene der Genehmigungserteilung angedeutet: Ist eine Ausführung und Abrechnung von Leistungen unter Verwendung der Genehmigung unter Berücksichtigung der Fachgebietsgrenzen völlig ausgeschlossen, kommt eine Erteilung bereits nicht in Betracht.

\section{Die Bestimmung der}

\section{Fachgebietsgrenzen anhand der Weiterbildungsordnung im Einzelfall}

Die Grenzen der fachärztlichen Berufsausübung bestimmen sich nach der Definition des Gebietes, zu dem die die jeweilige Facharztbezeichnung der (für den Facharzt geltenden) Weiterbildungsordnung zugeordnet werden kann (vgl. § 2 Abs. 2 S. 2 MWBO 2018), wobei ergänzend die einschlägigen Weiterbildungsinhalte heranzuziehen sind. Wie das BSG bereits mit Urteil vom 04.05.2016 $6^{31}$ herausgestellt hat, sind die Fachgebietsgrenzen dabei unabhängig von den individuellen Fähigkeiten des einzelnen Arztes, - auch von etwaigen Schwerpunktund Zusatzbezeichnungen, die der Arzt nach der Weiterbildungsordnung zu führen berechtigt ist, - zu beurteilen. ${ }^{32}$

Zu der Frage, welche Fassung der jeweiligen Weiterbildungsordnung der Landesärztekammern zur Bestimmung der Fachgebietsgrenzen heranzuziehen ist, hat das BSG folgende Festlegungen getroffen:

Maßgeblich für die Bestimmung der Fachgebietsgrenzen ist demnach grundsätzlich die jeweils aktuelle Fassung der Weiterbildungsordnung der für den jeweiligen

31 Vgl. BSG, Urteil vom 04.05.2016, а. а. O. (Fn. 2).

32 BSG, Urteil vom 04.05.2016, а. а. O. (Fn. 2), Rn. 23.
Vertragsarztsitz zuständigen Ärztekammer. ${ }^{33}$ Abzustellen sei hingegen nicht etwa auf jene Weiterbildungsordnung, die im Zeitpunkt der Facharztanerkennung Gültigkeit besa $\aleph^{34}$, ebenso nicht auf diejenige, die zum Zeitpunkt der Approbation des Arztes galt ${ }^{35}$. Zur Begründung führt der Senat aus:

„Das Abstellen auf die jeweils, alte‘ WBO hätte zur Folge, dass jedem Arzt seine, individuelle Fachgebietsgrenze' zuzuordnen wäre, je nachdem, aufgrund welcher WBO im Bereich welcher Landesärztekammer er seine Facharztanerkennung absolviert hat (vgl SG Berlin aaO; Schiller, MedR 2017, 182, 184). Zudem wären dann, wenn sich ein Fachgebiet durch die Implementierung neuer Untersuchungs- und Behandlungsmethoden weiterentwickelt, ältere Ärzte von der Erbringung und Abrechnung dieser Leistungen ausgeschlossen. “36

\section{Fazit}

Im Ergebnis ist festzuhalten, dass der Erteilung einer qualifikationsgebundenen $\mathrm{Ab}$ rechnungsgenehmigung nach einer Qualitätssicherungsvereinbarung, die auf der Grundlage von $\S 135$ Abs. 2 SGB V erlassen worden ist, eine etwaige Fachgebietsfremdheit der darauf bezogenen Leistung für den jeweiligen Facharzt grundsätzlich nicht entgegensteht.

Die Gebietsgrenzen des jeweiligen Fachgebietes bestimmen sich nach der Definition des Gebietes der Weiterbildungsordnung, dem der Facharzt zugerechnet werden kann (vgl. § 2 Abs. 2 S. 2 MWBO 2018), wobei individuelle Fähigkeiten des Arztes nicht zu berücksichtigen sind. Hinsichtlich der Inhalte des Fachgebietes ist grundsätzlich die aktuell geltende Fassung der Weiterbildungsordnung der für den Vertragsarztsitz des Arztes zuständigen Landesärztekammer heranzuziehen.

Im Falle eines Facharztes, der einem organbezogenen Fachgebiet zuzuordnen ist,

33 BSG, Urteil vom 15.07.2020, а. а. O. (Fn. 18), Rn. 20.

34 Ebd.

35 Vgl. BSG, Urteil vom 04.05.2016, a. a. O. (Fn. 2), Rn. 22.

36 BSG, Urteil vom 15.07.2020, а. а. O. (Fn. 18), Rn. 20. kann eine qualifikationsgebundene Abrechnungsgenehmigung für solche (fachübergreifenden) Leistungen erteilt werden, die für das Gebiet typischerweise fachfremd sind, wenn eine Erbringung dieser Leistungen in besonderen Fällen nicht ausgeschlossen ist. Das gilt auch im Falle von Fachärzten, die einem sog. Querschnittsfach zuzuordnen sind, also weder eindeutig einem Organ- noch eindeutig einem Methodenfach zuzuordnen sind. Die Frage nach einer etwaigen Fachfremdheit der Leistung für den Facharzt ist aber im Einzelfall Gegenstand des Verfahrens zur Prüfung der einzelnen Abrechnung durch die zuständige KV mit ggf. sich anschließender sachlich-rechnerischer Richtigstellung gemäß § 106 d SGB V.

Die Verweigerung einer Abrechnungsgenehmigung als Folge der Fachfremdheit der Leistung - selbst wenn der Arzt alle Anforderungen an die fachliche Befähigung erfüllt, die in der einschlägigen Qualitätssicherungsvereinbarung normiert sind - ist jedoch zulässig, wenn ein Gebrauch der Genehmigung durch den Facharzt im Vorhinein bereits ausgeschlossen ist. Dies ist der Fall, wenn der Facharzt einem Methodenfach zuzuordnen ist und die qualifikationsgebundene Abrechnungsgenehmigung sich auf Leistungen bezieht, die ausschließlich einem anderen Methodenfach zuzuordnen sind.

Erforderlich ist jedoch, insbesondere hinsichtlich sog. Doppelfachärzte, in jedem Fall, dass die Leistungen, auf die sich die qualifikationsgebundene Abrechnungsgenehmigung bezieht, auch dem Fachgebiet zugehörig sind, für das der Arzt vertragsärztlich zugelassen ist.

Prof. Dr. Peter Wigge

Rechtsanwalt

Fachanwalt für Medizinrecht

Philip Steuwer

Rechtsanwalt

Rechtsanwälte Wigge

Scharnhorststraße 40

48151 Münster

Telefon: (0251) 53 595-0

Telefax: (0251) 53 595-99

E-Mail: kanzlei@ra-wigge.de

www.ra-wigge.de 